\title{
Effects of Amphetamine on Sensorimotor Gating and Neurocognition in Antipsychotic-Medicated Schizophrenia Patients
}

\author{
Neal R Swerdlow*,', Savita G Bhakta', Jo A Talledo', Daniel M Franz', Erica L Hughes', Brinda K Rana' and \\ Gregory A Light' \\ 'Department of Psychiatry, UCSD School of Medicine, La Jolla, CA, USA
}

\begin{abstract}
Prepulse inhibition (PPI) of startle is being explored both as an indicator of target engagement for, and a biomarker predicting the sensitivity to, procognitive effects of drugs. We now report the effects of the pro-attentional drug, d-amphetamine, on PPI and neurocognition in antipsychotic-medicated schizophrenia patients and healthy subjects (HS) who were also tested in a targeted cognitive training (TCT) module. $44 \mathrm{HS}$ and 38 schizophrenia patients completed a double-blind, placebo-controlled crossover study of the effects of a single dose of amphetamine ( $10 \mathrm{mg}$ po) on PPI and MATRICS Consensus Cognitive Battery (MCCB) performance; TCT results were previously reported from 60 of these subjects. Moderators predicting AMPH sensitivity were assessed, including the rs4680 single-nucleotide polymorphism for catechol-O-methyltransferase (COMT). After placebo, patients exhibited PPI deficits with 60 ms prepulse intervals; these deficits were 'rescued' by amphetamine. The magnitude of amphetamine-enhanced PPI was greater in patients than in HS ( $p<0.032)$, and was associated with positive symptoms ( $p<0.007)$, antipsychotic load $(p<0.015)$, hedonic effects of AMPH ( $p<0.003)$, and with the presence of at least one methionine allele in rs4680 ( $p<0.008)$. No significant effects of amphetamine on MCCB performance were detected in either group, though pro-attentional effects of amphetamine in patients were associated with greater amphetamine-enhanced TCT learning. Amphetamine acutely 'normalized' PPI in antipsychotic-medicated schizophrenia patients; no concurrent acute neurocognitive changes were detected by the MCCB. Findings suggest that in the context of appropriate antipsychotic medication, a low dose of amphetamine enhances brain processes associated with higher function in schizophrenia patients, without accompanying changes in MCCB performance.
\end{abstract}

Neuropsychopharmacology (20I8) 43, 708-7|7; doi:I0.1038/npp.2017.285; published online 13 December 2017

\section{INTRODUCTION}

The experimental medicine strategy for identifying novel psychotherapeutics is facilitated by objective, quantitative laboratory-based measures that are impaired in patient groups, sensitive to drug effects, and regulated by identifiable brain circuitry of relevance to common psychiatric disorders. If these deficient measures are corrected in patients by acute drug treatment, this provides particularly powerful evidence for relevant 'target engagement'. Sensorimotor gating of startle, measured by prepulse inhibition (PPI), is regulated by disorder-relevant forebrain circuitry (cf. Swerdlow et al, 2001, 2008), and is impaired in several major psychiatric disorders, including schizophrenia (SZ) (Braff et al, 1978). In some studies, higher levels of PPI are associated with elevated cognitive and global function (Bitsios et al, 2006;

* Correspondence: Dr NR Swerdlow, Department of Psychiatry, UCSD School of Medicine, 9500 Gilman Drive, La Jolla, CA 92093-0804, USA, Tel: +619-543-6270, Fax: +619-543-2493,

E-mail: nswerdlow@ucsd.edu

Received 17 August 2017; revised 18 October 2017; accepted 8 November 2017; accepted article preview online 20 November 2017
Swerdlow et al, 2006a). Thus, PPI is being explored for its potential utility in experimental medicine approaches to psychotherapeutic development.

In the search for novel 'pro-cognitive' agents, one 'known' or reference drug for attention-enhancing effects is the psychostimulant, amphetamine. Acutely, amphetamine and other psychostimulants are known to have positive effects on specific neurocognitive measures in both healthy subjects (HS) and in individuals with a variety of disabling brain conditions including, among others, attention-deficit hyperactivity disorder (Rapoport et al, 1980), SZ (Barch, 2010; Barch and Carter, 2005; Goldberg et al, 1991; Pietrzak et al, 2010), and traumatic brain injury (Kim et al, 2006; McDonald et al, 2017). Interestingly, procognitive effects of amphetamine $(20 \mathrm{mg}, \mathrm{po})$ were not detected among a large cohort of adult HS using the MATRICS Consensus Cognitive Battery (MCCB), a gold standard for detecting procognitive drug effects (Nuechterlein et al, 2008); however, subgroups characterized by low baseline MCCB scores, higher age, and specific personality dimensions did exhibit amphetamineassociated gains in MCCB performance (Chou et al, 2013). 
We recently reported that amphetamine $(10 \mathrm{mg}$, po) acutely enhanced simple auditory learning in both HS and antipsychotic-medicated SZ patients, using a frequency modulation 'sound sweeps' task that is one component of a targeted cognitive training (TCT) program for this disorder (Swerdlow et al, 2016b); enhanced TCT learning among SZ patients after acute amphetamine was retained for at least one week. Here, we report on the effects of amphetamine on PPI and neurocognition in an extended sample of HS and antipsychotic-medicated SZ patients who had also been tested in the 'sound sweep' training program. PPI is regulated by both ventral forebrain and prefrontal dopamine fields (cf. Swerdlow et al, 2008), where amphetamine is known to exert dopamine-releasing effects. Since our previous findings suggest that amphetamine elevates PPI among subjects with low PPI baselines, we predicted that amphetamine would elevate PPI in antipsychotic-medicated SZ patients under stimulus conditions that elicited PPI deficits in these patients. Parallel studies in rodents were used to examine interactions of amphetamine with first- and second-generation antipsychotics in the regulation of PPI.

Two more specific predictions were tested in this experiment. First, we previously reported that PPI deficits in antipsychotic-medicated schizophrenia patients are most prominent with $60 \mathrm{~ms}$ prepulse intervals (Swerdlow et al, 2006a, 2014, 2017); thus, we predicted that among antipsychotic-medicated patients in the present study, PPI deficits would be most robust at this $60 \mathrm{~ms}$ interval, and that these $60 \mathrm{~ms}$ PPI deficits would be opposed by amphetamine. Second, because neurocognitive and behavioral sensitivity to amphetamine is reported to be moderated by catechol-Omethyltransferase (COMT; Hamidovic et al, 2010; Mattay et al, 2000, 2003), this experiment explicitly tested the prediction that COMT function-regulated by the COMT singlenucleotide polymorphism (SNP) rs4680-might moderate the sensitivity to amphetamine effects on PPI and/or MCCB performance. Some published literature suggests that rs 4680 valine homozygotes are more sensitive to the procognitive effects of amphetamine compared to methionine homozygotes (Mattay et al, 2000, 2003); however, we previously detected the opposite pattern when assessing amphetamine effects on TCT learning (Swerdlow et al, 2016b).

\section{MATERIALS AND METHODS}

This study was approved by the UCSD Human Subject IRB. Subjects were recruited via public advertisements and were paid for their participation. Written informed consent was obtained from all subjects.

Methods are similar to those described in Swerdlow et al (2016b). The present study sample $(n=82)$ is inclusive of all subjects $(n=60)$ reported in Swerdlow et al (2016b), with 22 additional subjects tested subsequent to completion of that report; none of the key dependent measures described in this report (startle, PPI, MCCB, and so on) were reported previously.

Participants ( $n$ 's: HS $=44$; schizophrenia $=38$; Table 1 ) were carefully screened to establish diagnoses and rule out exclusionary conditions (Supplementary Table S1). Patients reported being on stable antipsychotic doses for $>1$ month. After passing a phone- or in-person interview (assessing current and past medical and psychiatric history, medication
Table I Subject Characteristics

\begin{tabular}{|c|c|c|c|}
\hline Diagnoses (n) & $\mathrm{SZ}^{\mathrm{a}}(\mathbf{3 8})$ & HS (44) & $p$ \\
\hline Age in years (mean (SD)) & $40.4(7.9)$ & $29.7(11.2)$ & $<0.000$ । \\
\hline Weight in Ibs (mean (SD)) & $203.7(44.2)$ & I56.2(35.1) & $<0.000$ । \\
\hline $\operatorname{Sex}(M: F)$ & $21: 17$ & $32: 12$ & NS \\
\hline Smoker:nonsmoker & | 8:20 & $2: 42$ & $<0.000$ । \\
\hline Race (\% white) & $31.6 \%$ & $43.2 \%$ & NS \\
\hline Daily caffeine (mg) & $243.7(223.1)$ & $117.6(|5| .2)$ & $<0.0001$ \\
\hline $\begin{array}{l}\text { Wide range achievement Test } \\
\text { (WRAT) }\end{array}$ & $93.1(8.4)$ & $103.0(\mid 0.7)$ & $<0.000$ । \\
\hline Education (y; SD) & $12.2(1.8)$ & I5.0 (I.7) & $<0.0001$ \\
\hline Duration illness (y; SD) & | $8.9(8.5)$ & & \\
\hline Age of onset (y; SD) & $21.6(7.7)$ & & \\
\hline GAF (mean (SD)) & $63.9(8.2)$ & & \\
\hline
\end{tabular}

$\begin{array}{lc}\text { PANSS score (mean (SD)) } & \\ \text { Positive } & 20.9(4.3) \\ \text { Negative } & 20.5(3.9) \\ \text { Psychopathy } & 45.2(7.5) \\ \text { Total } & 86.6(12.7) \\ \text { Chlorpromazine equivalents (mg) } & 457.4(406.1) \\ \text { Anticholinergic load (pmol/mL } & 11.9(24.5) \\ \text { (SD)) } & \end{array}$

${ }^{a}$ Schizophrenia $(n=35)$, schizoaffective disorder, depressed $(n=3)$.

and recreational drug use, and family history of psychosis), subjects came to the laboratory (for women, within $72 \mathrm{~h}$ of menses onset) for the screening visit. During the screening visit, subjects were informed of the potential risks and benefits of the study, read and signed a consent form for study participation, underwent a screening medical interview, a structured diagnostic assessment (Mini International Neuropsychiatric Interview (M.I.N.I plus 6) for patients), physical examination and electrocardiogram, and completed urine toxicology with exclusion for any recreational drug; women underwent a urine-based pregnancy test. Audiometry confirmed hearing threshold $<40 \mathrm{~dB}(\mathrm{~A})$ at $1000 \mathrm{~Hz}$.

To most efficiently test an effect of COMT genotype on amphetamine sensitivity, DNA was first obtained from saliva collected from community samples of HS and schizophrenia patients (see Supplementary Methods) to identify rs4680 homozygotes (Val/Val: 'GG' or Met/Met: 'AA'), who were then studied to permit an efficient assessment of this SNP as a moderator of amphetamine sensitivity. Heterozygous (AG) individuals were initially 'waitlisted' for testing; when preliminary analyses demonstrated a significant effect of a genotype, groups were balanced by preferentially testing waitlisted AG (in addition to other later-identified GG and AA) subjects, to determine whether AGs demonstrated an intermediate $v s$ extreme phenotype; racial distributions are reported in Supplementary Results.

Test days (on which placebo or amphetamine were administered) included measures of startle, MCCB, subjective, and autonomic drug effects; performance on a targeted cognitive training 'sound sweeps' measure was reported previously (Swerdlow et al, 2016b). Startle 
measurement at the screening visit was used to confirm an adequate reflex magnitude to proceed with testing, as previously described (eg, Swerdlow et al, 2006a). The screening and test day startle sessions were identical. Broadband noise $(70 \mathrm{~dB}(\mathrm{~A}))$ preceded active stimuli by $3 \mathrm{~min}$ and persisted as a background noise during the test; this 3 min was followed by 42 trials, with six conditions: a $118-\mathrm{dB}(\mathrm{A})$ 40-ms noise burst (pulse alone) and the same burst preceded $10,20,30,60$, and $120 \mathrm{~ms}$ by a noise prepulse $16 \mathrm{~dB}$ above the background. Using $16 \mathrm{~dB}$ noise prepulses with this startle system, prepulse-associated EMG activity is $<0.5 \%$ of startle stimulus-induced levels (Swerdlow et al, 2006a). To measure startle habituation, three pulse-alone trials were presented at the session beginning (Block 1) and end (Block 3). Atypical AP's normalize PPI in SZ patients (cf. Swerdlow et al, 2008), but previous reports using startle equipment and methods comparable to those in this study identified small-to-medium-effect size deficits in PPI with $60 \mathrm{~ms}$ prepulse intervals, even among SZ patients taking atypical APs (Swerdlow et al, 2006a, 2014, 2017).

The MCCB was developed to evaluate neurocognition in trials of procognitive therapies for SZ and is accepted by the FDA as a primary end point (Nuechterlein et al, 2008). It measures seven key domains relevant to cognitive deficits in SZ and includes 10 tests that assess the speed of processing (SP), attention/vigilance (AV), working memory (WM), verbal learning (VL), visual learning (VsL), reasoning and problem solving (RP), and social cognition (SC), and provides $T$-scores for each domain and a composite score.

Subjects who passed the screening criteria were tested approximately 7 and 14 days later. Testing was double blind, and drug order was randomized. Blinding was achieved using pills that were repackaged by the hospital study pharmacist, and coded such that pill identity was not known to experimenters or study subjects. Test days were identical to screening days except (1) a pill administered $40 \mathrm{~min}$ prior to PPI (based on timecourse studies in HS (Swerdlow et al, 2002)) and $210 \mathrm{~min}$ prior to the MCCB was either placebo or amphetamine (10 mg), and 2) alternate forms of specific MCCB subtests (HVLT, BMVT) were used to blunt practice effects. This amphetamine dose (10 mg, po) and post-pill test time are reported to enhance neurocognition in SZ patients (Pietrzak et al, 2010).

On test days, subjects arrived at 0830 hours, ate a standardized breakfast, repeated audiometry, urine toxicology (and pregnancy testing in women), and a pill was administered at 0900. Heart rate (HR) and blood pressure (BP) were determined (sitting position, brachial cuff), and subjects completed a set of symptom-rating visual analog scales (VAS) at designated intervals that avoided test interruptions, starting before pill ingestion. HR, BP, and VAS scores across the post-pill intervals were thus anchored by a prepill baseline value. VAS scores assessed general somatic and psychological symptoms and level of consciousness (modified from Bond and Lader (1974) and Norris (1971)). Ratings assessed several states, but analyses were limited to the following three: 'happy', 'drowsy', and 'anxious'. Details of these rating scales are found in Swerdlow et al (2002). Autonomic and subjective VAS ratings were continued until $430 \mathrm{~min}$ post pill. Subjects were paid upon completion of each visit.

Supplementary studies of PPI in Long Evans rats were used to test potential interactions of amphetamine with different types of dopamine receptor antagonists, as a way to understand the mechanisms for observed amphetamine effects in SZ patients; this rat strain was selected based on ('human-like') relatively low sensitivity to PPI-disruptive effects of amphetamine (Talledo et al, 2009), as well as high levels of COMT gene expression, both in peripheral blood and forebrain and limbic structures, compared to other common outbred rat strains (eg, Sprague Dawley) (Swerdlow et al, 2012). The study design approximated that for startle testing in humans. Briefly, male Long Evans rats (250-300 g) were housed, handled, and baseline tested as per our published protocol (Shilling et al, 2008). Approximately 7 and 14 days later, PPI was assessed in each rat using startle stimuli identical to those described above for human testing, 10 min after administration of amphetamine (saline vehicle or $4.5 \mathrm{mg} / \mathrm{kg}, \mathrm{sc}$ ) in a balanced, within-subject crossover design. A set number of minutes prior to testing, rats were pretreated with one of several doses of the 'typical' antipsychotic haloperidol $(0.01,0.03$, or $0.1 \mathrm{mg} / \mathrm{kg}, \mathrm{sc})$, the atypical antipsychotic, quetiapine $(2.5,5.0$, or $7.5 \mathrm{mg} / \mathrm{kg}, \mathrm{sc})$, or the D1 antagonist, SCH $23390(0.01,0.03$, or $0.1 \mathrm{mg} / \mathrm{kg}$, sc); pretreatment was fixed for each rat across tests, and thus served as a between-subject factor. For detailed methods of drug preparation and administration, testing, and data analysis, please see Swerdlow et al (2005, 2006b, 2012). Findings are described in Supplementary Results.

For statistical analyses of human data, repeated measure ANOVAs identified the main and interaction effects of diagnosis (HS vs SZ) and dose (placebo vs $10 \mathrm{mg}$ ) on the dependent measures. These ANOVAs (main effects and twoand three-way interactions) were used to test the primary hypotheses (eg, amphetamine will enhance $60 \mathrm{~ms}$ PPI and MCCB performance in patients). Startle magnitude, latency, and \%PPI were averaged across right and left eyes, treated as continuous measures, and analyzed with repeated measure analyses of variance (ANOVAs) with appropriate post hoc comparisons. Startle measures were analyzed only in startle 'responders', ie, subjects for whom mean startle magnitude on pulse-alone trials was $\geqslant 10$ units $(1.31 \mu \mathrm{V} /$ unit; Swerdlow et al, 2006a, 2014, 2017). Based on known sex differences in PPI (Swerdlow et al, 1993), sex was included as a between factor in all primary analyses of PPI. Post hoc analyses assessed specific effects of variables (eg, COMT genotype) on the primary outcomes. Once ANOVAs detected significant effects of amphetamine on PPI, post hoc exploratory correlations were assessed among changes in PPI and neurocognition, and subjective and autonomic drug responses and clinical variables. MCCB $T$-scores were also analyzed via ANOVAs, with diagnosis as a between- and drug, test order and cognitive domain as within factors. Based on known effects of amphetamine on attention, $a$ priori post hoc analyses of amphetamine effects on the MCCB attention/vigilance domain were pursued.

To assess the subjective effects of amphetamine, VAS scores in $\mathrm{mm}$ were first reduced by subtracting prepill values from the value at each post-pill time point. To calculate the magnitude of the amphetamine effect, a difference score (amphetamine minus placebo values) was then calculated for each post-pill time point, and treated as a continuous measure for regression analyses. Data for the subjective and autonomic effects of amphetamine from the majority $(n=60)$ of the present subjects have been previously 
reported (Swerdlow et al, 2016b). Alpha for planned comparisons and empirical findings was set at 0.05 and 0.01 , respectively. Additional descriptive statistics are reported for demographics, autonomic, and subjective measures not directly linked to a specific hypothesis.

\section{RESULTS}

\section{Subject Characteristics}

As seen in Table 1, patients were functionally impaired, symptomatic, and taking second-generation antipsychotics; in addition to antipsychotics, common ( $>10 \%)$ medication classes included antidepressants (37\%), anticholesterol medication (26\%), mood stabilizer/ anticonvulsants (24\%), antihypertensives (24\%), anticholinergics (24\%), soporifics (18\%), antidiabetics (11\%), and benzodiazepines (11\%). Patient vs HS differences were detected in age, education, premorbid intelligence, and smoking status.

\section{Amphetamine Bioactivity}

Analyses revealed the expected positive chronotropic and pressor effects of amphetamine, evident shortly after oral administration and lasting throughout the test day
(Supplementary Figure S1); these effects were blunted in SZ patients, as previously reported (Modell and Hussar, 1965). Compared to placebo, amphetamine diminished the drowsiness that developed across the test day, though this effect was also blunted in patients; consistent with past reports (Hamidovic et al, 2010), amphetamine effects on drowsiness were moderated by rs4680 (amphetamine $\times$ genotype: $\mathrm{F}=3.80$, df $2,72, p<0.03)$, being more robust among rs4680 Val homozygotes than Met homozygotes $(\mathrm{p}<0.01)$ or Val/Met heterozygotes $(p<0.04)$ (Supplementary Figure S1). About $3 \mathrm{~h}$ post pill, 'happy' scores were elevated after amphetamine $v s$ PBO, independent of diagnosis (Supplementary Figure S2). Despite this bioactivity, only $57 \%$ of all HS and $50 \%$ of all patients correctly identified the active drug day.

\section{Startle Reflex}

No significant effects of diagnosis or amphetamine were detected on measures of pulse-alone startle reflex magnitude or reflex habituation (all F's $<1$; Figure 1a and b). Analysis of peak startle latency revealed arithmetic but not statistically significant slowing in patients $v s \mathrm{HS}(\mathrm{F}=3.04$, df $1,56, \mathrm{NS})$, with no effect of amphetamine $(\mathrm{F}<1)$, and no interaction of diagnosis $\times$ amphetamine $(\mathrm{F}<1)$ (Figure 1c). The expected
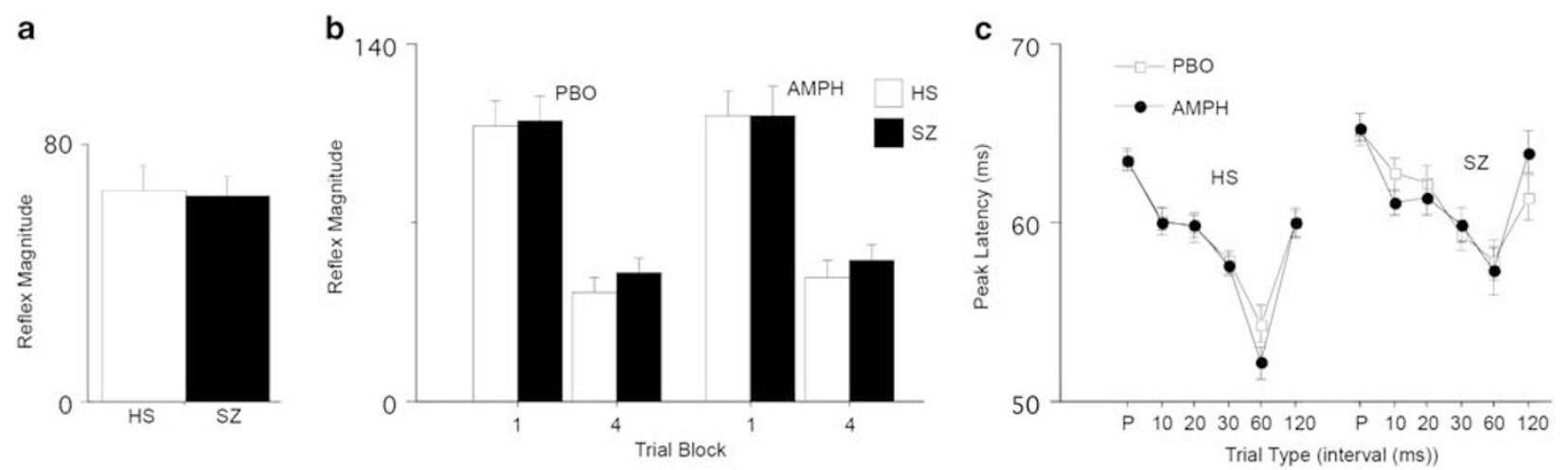

Figure I Startle reflex magnitude (a) and habituation (b) on pulse-alone trials in HS and schizophrenia (SZ) patients detected no main or interaction effects of diagnosis and amphetamine (AMPH) vs placebo (PBO). Peak reflex latency measures (c) detected known effects of trial type (facilitation by prepulses) and diagnosis (slowing in SZ patients), but no main or interaction effects of amphetamine.
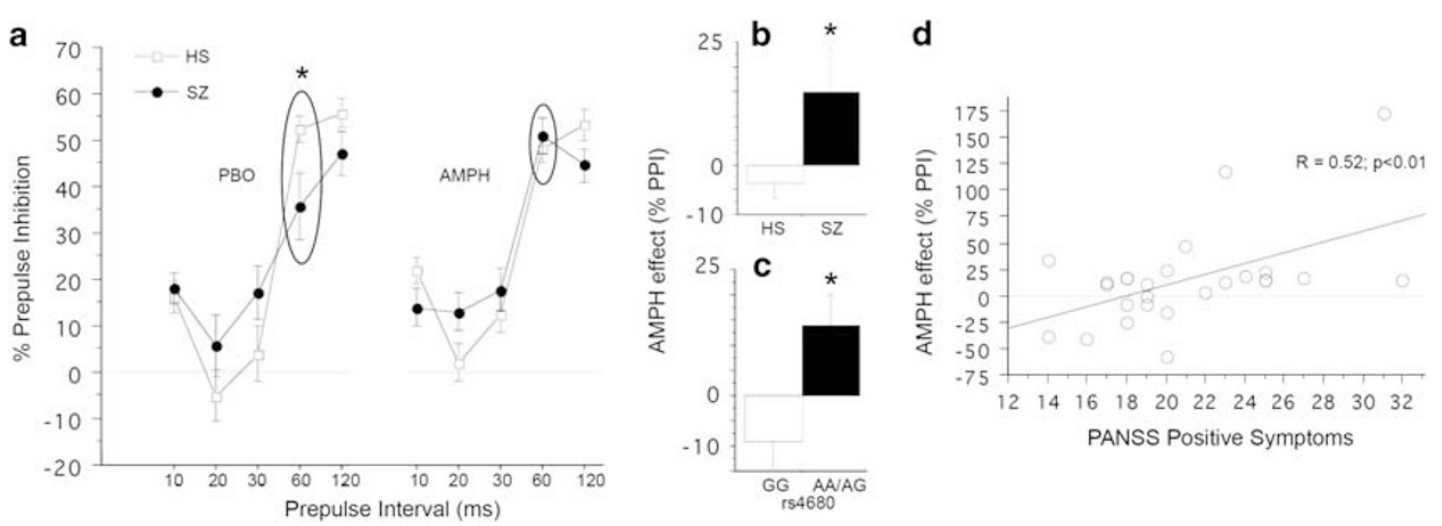

Figure 2 (a) \%PPI across 10-120-ms prepulse intervals after placebo (left) and amphetamine (right) in HS and patients. After placebo (left), patients exhibited previously reported PPI deficits at 60 ms prepulse intervals (indicated by a large oval; $*=0.05 ; d=0.45$ ); amphetamine increased \%PPI at 60 ms intervals in patients but not HS (right), 'rescuing' the PPI phenotype. The amount of amphetamine-enhanced PPI was greater in patients vs HS (b; * $p<0.032$ ), and among subjects with vs without a methionine allele at rs4680 (c; * $<0.008)$, and correlated significantly with the level of PANSS-positive symptoms (d; $p<0.015)$. 
facilitation of latency by prepulses was evident in a significant effect of trial type $(\mathrm{F}=48.45$, df 5,28 , $p<0.0001$ ), but there was no significant interaction of trial type with amphetamine $(\mathrm{F}=2.11$, df 5,280 , NS), nor a significant three-way interaction with diagnosis $(\mathrm{F}<1)$.

Analysis of \%PPI (Figure 2a) revealed no significant main effects of diagnosis $(\mathrm{F}<1)$, amphetamine $(\mathrm{F}=1.81$, df 1,59 , $\mathrm{NS})$, or sex $(\mathrm{F}<1)$, but there were significant 2 - and 3-way interactions of diagnosis $\times$ interval $(\mathrm{F}=4.00$, df 4,236 , $p<0.004)$ and diagnosis $\times$ interval $\times$ amphetamine $(\mathrm{F}=3.08$, df $4,236, p<0.02)$, and a near-significant interaction of diagnosis $\times$ interval $\times \operatorname{sex}(F=3.92, \mathrm{df} 1,59, p<0.053)$. Based on our past reports of small-to-medium-effect size PPI deficits in antipsychotic-medicated SZ patients at $60 \mathrm{~ms}$ prepulse intervals, post hoc comparisons at this interval confirmed a significant diagnosis $\times$ amphetamine interaction $(\mathrm{F}=4.13$, df $1,59, p<0.05)$, with reduced PPI among patients $v s$ controls after placebo $(\mathrm{F}=3.97, \mathrm{df} 1,61, p=0.05$; $d=0.45)$, but not after amphetamine $(\mathrm{F}<1)$. The magnitude of the 'amphetamine effect' on PPI (amphetamine minus placebo) was significantly greater for patients $(+14.85 \%) v s$ HS $(-3.68 \%)(\mathrm{F}=4.89$, df $1,61, p<0.032)$ (Figure $2 \mathrm{~b})$. This was also true in age range-matched subgroups of patients and HS (age range: $23-55$ y; age, patient $v s \mathrm{HS}: \mathrm{F}=1.92$, df 1 , 46, NS; amphetamine effect on PPI: SZ $>\mathrm{HS}, \mathrm{F}=4.67$, df 1 , $46, p<0.04)$. We previously reported that the PPI-enhancing effects of the NMDA antagonist, memantine, in SZ patients were significantly associated with age, being most robust in older patients (Swerdlow et al, 2016a); this was not the case with amphetamine in the present study $(r=-0.08$, NS).

Demographic and clinical correlates of PPI amphetamine sensitivity are seen in Table 2 . The rs4680 polymorphism for COMT was specifically assessed, based on controversies related to its role in moderating amphetamine effects on neurocognition (Mattay et al, 2003; Hamidovic et al, 2010; Hart et al, 2013), and our recent findings suggesting the greatest amphetamine-enhanced TCT learning among 'Met/ Met' HS and SZ patients, all of whom were included within the present sample (Swerdlow et al, 2016b). Analyses of the 'amphetamine effect' for $60 \mathrm{~ms}$ prepulse intervals, using rs4680 genotype as a grouping factor, revealed a significant main effect of a genotype $(\mathrm{F}=3.14, \mathrm{df} 2,57, p=0.05)$, but no significant interaction of diagnosis $\times$ genotype. Opposite to the genotype effects on amphetamine-reduced 'drowsiness' (above), but similar to those observed with TCT learning (Swerdlow et al, 2016b), the contrast of methionine $v s$ valine homozygotes (AA vs GG) revealed significantly greater sensitivity among 'AA' individuals $(\mathrm{F}=8.65, \mathrm{df} 1,41$, $p<0.006)$. Analyses comparing individuals carrying at least one methionine allele $v s$ 'GG' subjects yielded a similar result favoring methionine carriers $(\mathrm{F}=7.77$, df $1,61, p<0.008)$ (Figure 2c). Separate analyses among the four most represented racial backgrounds (White, African American, Asian, and Native/Alaskan American) confirmed the main effect of genotype $(\mathrm{F}=6.51$, df $2,41, p<0.004)$ and no significant race $\times$ genotype interaction (NS); arithmetically greater amphetamine effects among AA vs GG individuals were evident in each of these four racial groups.

Among the other variables assessed in exploratory analyses (Table 2), the only predictors of PPI amphetamine sensitivity that met the exploratory threshold of alpha (0.01) were PANSS-positive symptoms $(r=0.52, p<0.007)$ (Figure $2 \mathrm{~d}$ )
Table 2 Correlations ( $R$ ) vs AMPH-Enhanced 60 ms PPI in Schizophrenia Patients

\begin{tabular}{lcc}
\hline & $\boldsymbol{R}$ & $\boldsymbol{p}$ \\
\hline Age (y) & -0.08 & $\mathrm{NS}$ \\
GAF & 0.21 & $\mathrm{NS}$ \\
Duration illness (y) & 0.15 & $\mathrm{NS}$ \\
Chlorpromazine equivalents (mg) & 0.47 & $<0.015$ \\
Anticholinergic load' & $\mathrm{R}_{\mathrm{s}}=0.19$ & $\mathrm{NS}$ \\
PANSS positive & 0.52 & $<0.007$ \\
PANSS negative & 0.34 & $\mathrm{NS}$ \\
PANSS psychopathy & 0.27 & $\mathrm{NS}$ \\
PANSS total & 0.42 & $<0.032$ \\
AMPH-enhanced ANV & 0.08 & $\mathrm{NS}$ \\
AMPH-enhanced HRc & 0.15 & $\mathrm{NS}$ \\
AMPH-enhanced sBPc & 0.17 & $\mathrm{NS}$ \\
AMPH-enhanced dBPc & 0.15 & $\mathrm{NS}$ \\
AMPH-enhanced 'drowsiness'c & -0.02 & $\mathrm{NS}$ \\
AMPH-enhanced 'happycc & -0.59 & $<0.03$ \\
\hline
\end{tabular}

Abbreviations: dBP, diastolic blood pressure; GAF, Global Assessment of Functioning Scale; HR, heart rate; PANSS, Positive and Negative Symptom Scale; sBP, systolic blood pressure; WRAT, Wide Ranging Achievement Test.

${ }^{a}$ Anticholinergic load $(\mathrm{pmol} / \mathrm{mL}$ ) not normally distributed.

${ }^{b}$ Order-corrected amphetamine effect on MCCB attention/vigilance domain. Immediately after PPI testing.

and amphetamine-enhanced 'happy' ratings at the time of PPI testing $(r=-0.59, p<0.003)$. Antipsychotic dose may have moderated both of these measures, as greater chlorpromazine equivalents were positively associated with amphetamine-enhanced PPI $(r=0.47, p<0.015)$ and negatively associated with amphetamine-enhanced 'happy' ratings at the time of PPI testing $(r=-0.45, p<0.006)$.

Studies conducted in male Long Evans rats to understand the potential contribution of antipsychotic medications to the observed PPI-enhancing effects of amphetamine detected dose-dependent effects of haloperidol, quetiapine, and $\mathrm{SCH}$ 23390 on amphetamine-modified PPI, as described in the Supplementary Results (Supplementary Figure S2). In these studies, amphetamine significantly enhanced short-interval PPI (10-30 ms intervals), and modestly reduced long-interval PPI (60-120 ms intervals), after vehicle pretreatment. Pretreatment with haloperidol $(0.1 \mathrm{mg} / \mathrm{kg})$ increased $60 \mathrm{~ms}$ PPI in amphetamine-treated rats, and pretreatment with quetiapine $(7.5 \mathrm{mg} / \mathrm{kg})$ increased $30 \mathrm{~ms}$ PPI in amphetaminetreated rats. The PPI-enhancing effects of amphetamine in these rats appeared to require the availability of D1 receptors, since they were prevented by the D1 antagonist, SCH 23390 $(0.03 \mathrm{mg} / \mathrm{kg})$.

\section{МСCB}

ANOVA of cognitive domain T-scores (Figure 3a) revealed the expected main effect of diagnosis ( $\mathrm{HS}>\mathrm{SZ}: \mathrm{F}=53.05$, df $1,77, p<0.0001)$, with no main effects of amphetamine $(\mathrm{F}<1)$ or test order $(\mathrm{F}=1.68$, df $1,77, \mathrm{NS})$, but a significant interaction of amphetamine $\times$ test order $(\mathrm{F}=10.83$, df 1,77 , $p<0.002)$. There was a significant effect of MCCB domain $(\mathrm{F}=17.71$, df 6, 462, $p<0.0001)$, and significant interactions 


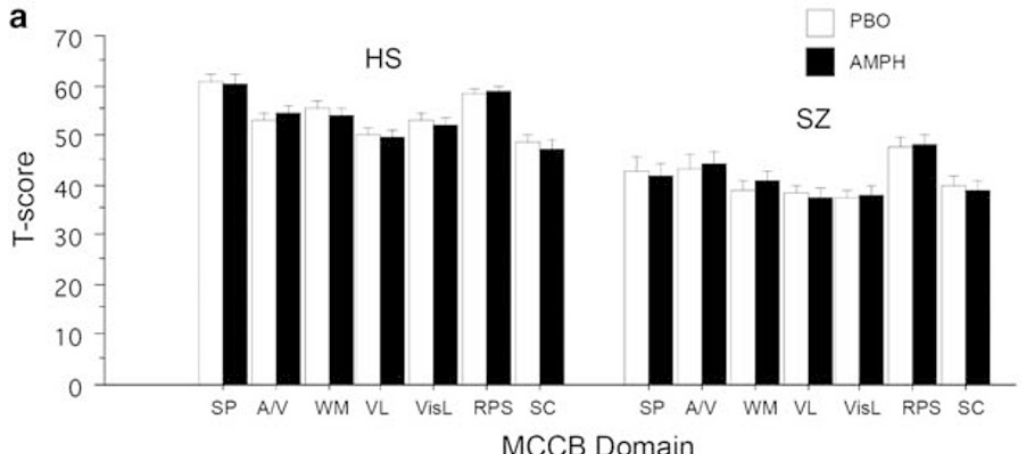

b

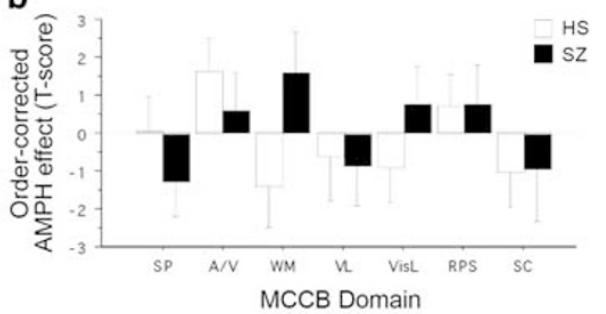

Figure 3 (a) MCCB T-scores in HS and patients, across seven MCCB domains (AN, attention/vigilance; RPS, reasoning and problem solving; SC, social cognition; SP, speed of processing; VisL, visual learning; VL, verbal learning; WM, working memory). Analyses confirmed significant MCCB deficits in patients vs HS, but no main or interaction effects of amphetamine. (b) Effect of amphetamine on T-scores in each domain, corrected for order effects. No significant effects of diagnosis were detected.

of domain $\times$ diagnosis $(\mathrm{F}=3.87, \mathrm{df} 6,462, p<0.002)$ and of domain $\times$ amphetamine $\times$ test order $(F=5.31$, df 6,462 , $\mathrm{p}<0.0001)$. Based on the known pro-attentional effects of amphetamine, post hoc analyses focused on the MCCB domain of Attention/Vigilance (A/V). ANOVA revealed the expected effect of diagnosis $(\mathrm{F}=15.07$, df $1,77, p<0.0002)$, no significant effect of amphetamine $(\mathrm{F}=2.73$, df 1,77 , $p=0.10$ ), and a significant interaction of amphetamine $\times$ test order $(\mathrm{F}=12.13$, df $1,77, p<0.0009)$. This interaction reflected the fact that subjects scored higher after amphetamine if it was administered on test day $2(p<0.001)$, but not if it was administered on test day 1 (NS).

To try to dissociate order and drug effects on $\mathrm{A} / \mathrm{V}$, we calculated an 'order-corrected' A/V value (Figure 3b), described previously (Bhakta et al, 2016). Briefly, the mean amphetamine effect on $\mathrm{A} / \mathrm{V}$ T-scores (amphetamine minus placebo) for each subject was adjusted by an amount equal to the group mean order effect on A/V T-scores (Day 2 minus Day 1). This order-corrected amphetamine effect (OCAE) (means $=+1.62$ and $+0.58 \mathrm{~T}$-score units for HS and patients, respectively) did not differ significantly based on diagnosis $(\mathrm{F}<1)$; in patients, it was arithmetically larger among men $v s$ women, smokers vs nonsmokers, rs4680 AA (+3.60) vs AG $(-0.05)$ and GG $(-0.54)$ subjects, and subjects with belowmedian $(+2.09)$ vs above-median $(-1.10)$ screen day A/V Tscores, but none of these differences reached statistical significance. Also, among patients, this OCAE value correlated significantly with amphetamine-enhanced TCT learning $(r=0.34, p<0.05)$ but not amphetamine-enhanced PPI at $60 \mathrm{~ms}$ intervals $(r=0.08, \mathrm{NS})$.

\section{DISCUSSION}

We recently reported that amphetamine (10 mg, po) significantly enhanced learning in antipsychotic-medicated schizophrenia patients, using an auditory frequency discrimination TCT task (Swerdlow et al, 2016b). Here, we report data from an extended sample of these subjects in which amphetamine 'normalized' $60 \mathrm{~ms}$ PPI among SZ patients, while not modifying PPI in HS; we previously discussed the potential importance of this $60 \mathrm{~ms}$ interval to the flow of preconscious information into conscious awareness in SZ (Grobstein, 2005; Kanabus et al, 2002; Libet et al, 1979; Libet, 1985; Swerdlow et al, 2006a). In contrast to TCT learning and PPI, MCCB performance was not significantly changed by amphetamine, though by correcting for test order, it was possible to demonstrate that amphetamineinduced changes in attention correlated modestly, but significantly, with gains in TCT learning. To our knowledge, this is the first report of amphetamine effects on PPI or MCCB performance in SZ patients. A review of the literature of these amphetamine effects in HS presents some interpretative challenges.

Hutchison and Swift (1999) first reported that amphetamine (20 mg, po) reduced PPI in healthy men and women. They noted that this effect was not moderated by sex, but later (Hutchison et al, 1999) reported that it was largely restricted to individuals with elevated scores of the novelty seeking (NS) Scale of Cloninger's (1987) Tridimensional Personality Scale (TPQ). In a small study of the time course of dopamine agonist effects in men, we (Swerdlow et al, 2002) failed to detect PPI-reducing effects of amphetamine (20 mg, po), but rather noted a modest increase in PPI at a time point (90 min post pill) where Hutchison and Swift (1999) had detected the opposite pattern. In a follow-up, larger study in men, we (Swerdlow et al, 2003) again failed to detect robust PPI-reducing effects of d-amphetamine $(20 \mathrm{mg}$, po), even among individuals with the highest scores on the TPQ NS Scale; PPI was modestly reduced by amphetamine only at prepulse intervals (10-20 ms) substantially shorter than those examined in earlier reports. This study did note an inverse relationship between baseline PPI levels and postamphetamine PPI levels, suggesting that individuals with high-baseline PPI levels were most sensitive to the PPIreducing effects of amphetamine, while individuals with lowbaseline PPI level were most likely to exhibit amphetamineenhanced PPI. Using an attention-modulated measure of PPI and doses of 5,10 , and $20 \mathrm{mg}$ amphetamine po in healthy men and women, Alessi et al (2003) reported no significant effects of amphetamine on PPI, though they did note lower PPI after amphetamine among individuals with higher Sensation Seeking Scale (SSS) scores (Zuckerman et al, 1978). In a study limited to healthy women (Talledo et al, 2009), we again failed to detect the overall effects of amphetamine (20 mg, po) on PPI, though we did replicate and extend our previous observation that amphetamine effects on PPI were dependent on baseline PPI levels (Swerdlow et al, 2003), as well as the findings by 
Hutchison et al (1999) and Alessi et al (2003) that amphetamine effects on PPI were moderated by both NS scores on the TPQ (Hutchison et al, 1999) and by SSS scores (Alessi et al, 2003). The moderating effects of baseline PPI level on PPI sensitivity to dopamine agonists were also confirmed by Bitsios et al (2005). Most recently, Chitty et al (2014) used an attention-modulated PPI paradigm with a range of pulse intensities, and detected negligible effects of amphetamine $(0.45 \mathrm{mg} / \mathrm{kg}$, po) on PPI. Preller et al (2013) reported elevated PPI levels associated with chronic cocaine use.

These studies differed in many ways, including test design ('instructed' (attentional) vs 'uninstructed' (preattentional) PPI), study sample (male $v s$ female $v s$ mixed sample), stimulus parameters (tone $v s$ noise burst, pulse intensity, prepulse intervals, and so on), testing times (60-150 min post pill), dose (5-34 mg, po), and independent measures (TPQ, SSS, and so on). On balance, however, the findings suggest that amphetamine effects on PPI in healthy humans are not statistically robust across an inclusive and biologically heterogeneous study cohort, but that subgroups of individuals-perhaps characterized by high- vs low-baseline PPI levels and/or specific putatively dopamine-linked personality indices-might be sensitive to either PPI-reducing or PPIenhancing effects of amphetamine. The present studies failed to detect significant effects of $10 \mathrm{mg}$ amphetamine on PPI in HS, but did detect PPI-enhancing effects of amphetamine in SZ patients restricted to the $60 \mathrm{~ms}$ prepulse interval, where PPI levels are deficient in these patients.

Speculation regarding the mechanism(s) by which amphetamine might enhance PPI in antipsychotic-medicated SZ patients is made challenging by many factors, including the lack of localizing information (eg, brain imaging), the heterogeneity of the medication regimens, and the presumed heterogeneous biology of SZ in the present sample. There is substantial evidence from laboratory animals, and modest evidence from humans, for a dopaminergic regulation of PPI (cf. Swerdlow et al, 2008). In very broad terms, prodopaminergic effects acting on subcortical (eg, ventral forebrain) D2 dopamine receptors are thought to reduce PPI, while in prefrontal dopamine fields, PPI disruption is associated with dopamine receptor blockade, particularly at D1 receptors (Swerdlow et al, 2001, 2005). Amphetamineenhanced PPI in SZ patients might reflect complex interactions between amphetamine and the heterogeneous regimens of antipsychotic medications in this study; our findings in rodents reveal dose-dependent effects of different dopamine receptor antagonists on amphetamine-modified PPI. Conceivably, antipsychotic medications that oppose subcortical dopamine-enhancing effects of amphetamine, but are less active against prefrontal D1-activating effects of amphetamine, might account for the observed PPI-enhancing effects of amphetamine in this study. Such a model is surely oversimplified-for example, it does not consider a potential role of SZ-related alterations in local circuitry and dopamine receptors-but might be testable using PPI in combination with neurochemical imaging strategies, similar to other investigations of amphetamine effects on forebrain dopamine activity in SZ patients (Slifstein et al, 2015).

Weafer et al (2017) recently integrated subjective, behavioral, and brain-activation measures after amphetamine (20 mg, po) and reported that HS with lower levels of behavioral inhibition and less activation in the right middle frontal gyrus during a reaction time task reported greater levels of amphetamine-induced euphoria. While the present study applied different metrics, we detected no relationship in HS between either baseline (screening) levels of PPI, or PPI amphetamine sensitivity, and amphetamine-induced increases in 'happy' VAS scores. Interestingly, in patients, we detected an inverse relationship between amphetamineincreased PPI and amphetamine-enhanced 'happy' scores; conceivably, greater amphetamine-induced euphoria in patients may reflect dopamine activation in ventral forebrain (and particularly, mesolimbic) regions associated with PPIdisruptive effects of amphetamine (cf. Swerdlow et al, 2001). Consistent with this hypothesis, at the time of PPI testing, greater antipsychotic load (and thus, presumably, greater forebrain D2 blockade) was associated both with less amphetamine-euphoria $(p<0.006)$, and with more amphetamine-enhanced PPI $(p<0.015)$. Clearly, given the lack of brain-imaging evidence, the relatively small sample size, heterogeneous medication regimens, and blunt metrics of 'euphoria', this hypothesis remains highly speculative.

Two observations deserve mention related to the potential mechanisms for these acute amphetamine effects. First, amphetamine effects on PPI-like those observed on TCT learning (Swerdlow et al, 2016b)-were evident (and more robust) in patients, despite the fact that amphetamine effects on autonomic measures and drowsiness were blunted in these patients. This pattern suggests that amphetamine's effects on PPI and TCT learning in these patients are not dependent on its ability to produce autonomic activation, or to reduce drowsiness. Second, while some effects of amphetamine appear to be most robust among rs4680 Val homozygotes (including, eg, its drowsiness-reducing effects in the present study), amphetamine's potentiating effects on PPI- and TCT learning in SZ patients appear to be most robust among rs4680 Met homozygotes. Historical precedence suggests caution in interpreting the mechanistic contributions of prefrontal COMT based on such findings with rs4680 (Hart et al, 2013; Mattay et al, 2003; Wardle et al, 2013). This dissociation nonetheless suggests that amphetamine's effects on PPI and TCT learning cannot be easily explained by changes in drowsiness, and moreover, that different mechanisms likely underlie amphetamine's effects on one (increased PPI and TCT learning, greatest in Met/ Met individuals) vs the other (reduced drowsiness, greatest in $\mathrm{Val} / \mathrm{Val}$ individuals) type of changes.

While many studies have reported pro-attentional effects of acutely administered amphetamine in HS and even SZ patients, evidence for such effects has not been generated using the MCCB, which is the 'gold standard' for detecting procognitive drug effects. We previously reported no robust effects of $20 \mathrm{mg}$ amphetamine on MCCB performance in 60 HS (Chou et al, 2013), though we could not rule out the possibility that these effects were obviated by high MCCB performance among a healthy sample of mostly young college students. In the present study, procognitive effects of amphetamine were not detected by the MCCB, even among SZ patients who were clearly not performing at 'ceiling' levels. Conceivably, the pro-attentional effects of amphetamine might have been blocked by antipsychotic medications in this sample; such an explanation would not be parsimonious, however, based on (1) previously reported 
procognitive effects of amphetamine in antipsychoticmedicated SZ patients (Barch and Carter, 2005; Goldberg et al, 1991; Pietrzak et al, 2010); (2) evidence for amphetamine-enhanced learning (Swerdlow et al, 2016b) and PPI (present study) in these same antipsychoticmedicated subjects.

A limitation of this study is the lack of 'matched' patient and control groups; these groups differ significantly across a number of variables, including age, caffeine use, and smoking status, among many others. Importantly, the key finding of this study did not rely on a comparison across patient $v s$ control groups, but rather involved a within-subject comparison of placebo $v s$ amphetamine effects on PPI in patients. This amphetamine effect on PPI was unrelated to age among patients; across groups, the magnitude of this amphetamine effect was greater in patients than controls, and this finding was also evident in age-matched subgroups of patients and controls. While neither comparisons survived the adjusted alpha for exploratory analyses (0.01), the magnitude of the amphetamine effect tended to be greater among smoker $v s$ nonsmoker patients $(p<0.10)$, and was positively associated with caffeine intake among patients $(p<0.02)$ but not controls (NS). These preliminary observations may warrant reexamination in future studies.

Detecting and interpreting acute cognitive effects of amphetamine (or other drugs, such as memantine (Bhakta et al, 2016)) in a within-subject crossover design present the major challenge of detecting a drug 'signal' on neurocognitive performance, above that of potent order effects. We have discussed this issue at some length (Bhakta et al, 2016; Chou et al, 2013). The present study addressed this challenge in three ways. First, different versions of the MCCB were used in each of the three MCCB tests (screen day, tests 2 and 3). Second, the greatest differences in practice effects are generally detected between the first and second test experiences; completing a 'screening' MCCB presumably blunted the impact of practice effects on performance during subsequent placebo $v s$ amphetamine days (tests 2 and 3 ). Third, an 'order corrected drug effect' was calculated, as in Bhakta et al (2016). We previously reported that, compared to the bimodal distribution of drug effects on MCCB performance on test days $1 v s 2$, this order-corrected value exhibits a normal, unimodal distribution; we also previously reported that this order-corrected value 'performs' as predicted, ie, it is moderated by variables in ways that are similar to other drug effects known to be relatively immune to order effects (eg, drug-altered PPI (Bhakta et al, 2016)). In the present study, the order-corrected amphetamine effect on A/V scores correlated significantly with amphetamineenhanced TCT learning in SZ patients. While this metric of drug-enhanced neurocognition is still far from ideal, the present data provide some ecological validity by demonstrating its relationship to function (learning)-enhancing drug properties in these patients.

The MCCB is intended to be sensitive to sustained changes in neurocognition in longer-term clinical trials, presumably based on higher-order integrative changes among distributed neural networks. In contrast, our experimental medicine approach aims to assess acute drug-induced changes in lower-level sensory and sensorimotor processing which are determinants of neurocognition and function in SZ patients (Thomas et al, 2017). These measures of sensory and sensorimotor function such as PPI, mismatch negativity $(\mathrm{MMN})$, auditory steady-state response (ASSR), and even auditory TCT learning have repeatedly shown acute changes after a single drug dose (eg, Light et al, 2017; Swerdlow et al, 2016b); conceivably, the MCCB may not be sensitive to the adaptive brain changes evoked by such acute drug challenges. Conversely, while higher levels of PPI, MMN, ASSR, and other neurophysiological measures of early sensory processing are sometimes associated with better cognitive performance and function in SZ patients and HS (Bitsios et al, 2006; Swerdlow et al, 2006a; Thomas et al, 2017), there is no current evidence that drug-induced increases in these measures result in cognitive or functional gains, or any clear understanding of the time course and conditions (eg, concomitant cognitive training) needed to produce such changes.

We detected no adverse effects of amphetamine administration in these 38 schizophrenia patients. Nonetheless, the present findings do not argue for the use of amphetamine or other stimulants to enhance cognition in SZ patients. These findings do, however, suggest that-as we have previously demonstrated with the NMDA antagonist, memantine (Swerdlow et al, 2016a) - brain mechanisms regulating sensorimotor gating in antipsychotic-medicated SZ patients maintain significant plasticity that can be accessed and enhanced via acute drug challenge. Taken together with our recent evidence for acute prolearning effects of amphetamine in a subset of these patients (Swerdlow et al, 2016b), there is a growing case for the use of pharmacological agents not simply to control the more disruptive psychotic symptoms of SZ, but also to tap into positive neuroplastic mechanisms underlying cognition and learning, in the service of learning-based therapies. The utility of psychostimulants in this capacity has been validated in clinical conditions with severe cognitive dysfunction, such as traumatic brain injury (McDonald et al, 2017); for neurocognitive and functional deficits in schizophrenia, such a role for psychostimulants or other putative pro-attentional drugs, remains largely untested (Swerdlow, 2011).

\section{FUNDING AND DISCLOSURE}

This work has been funded by the Brain \& Behavior Research Foundation through a Distinguished Investigator Award to NRS, and by the National Institutes of Mental Health through grants to NRS (R01-MH059803 and R01MH094320); and to SGB (1KL2TR001444). GAL has served as a consultant for Astellas, Boehringer Ingelheim, Merck, Lundbeck, Neuroverse, NeuroSig, and Takeda. The remaining authors declare no conflict of interest.

\section{ACKNOWLEDGMENTS}

Outstanding administrative support was provided by Ms. Maria Bongiovanni. Excellent technical support was provided by Ms. Alexis Alvarez.

\section{REFERENCES}

Alessi SM, Greenwald M, Johanson CE (2003). The prediction of individual differences in response to $\mathrm{D}$-amphetamine in healthy adults. Behav Pharmacol 14: 19-32. 
Barch DM (2010). Pharmacological strategies for enhancing cognition in schizophrenia. Curr Top Behav Neurosci 4: 43-96.

Barch DM, Carter CS (2005). Amphetamine improves cognitive function in medicated individuals with schizophrenia and in healthy volunteers. Schizophr Res 77: 43-58.

Bhakta S, Chou HH, Rana B, Talledo J, Balvaneda B, Gaddis L et al (2016). Effects of acute memantine administration on MATRICS Consensus Cognitive Battery performance in psychosis: Testing an experimental medicine strategy. Psychopharmacology 233: 2399-2410.

Bitsios P, Giakoumaki SG, Frangou S (2005). The effects of dopamine agonists on prepulse inhibition in healthy men depend on baseline PPI values. Psychopharmacology 182: 144-152.

Bitsios P, Giakoumaki SG, Theou K, Frangou S (2006). Increased prepulse inhibition of the acoustic startle response is associated with better strategy formation and execution times in healthy males. Neuropsychologia 44: 2494-2499.

Bond A, Lader M (1974). The use of analogue scales in rating subjective feelings. Br J Med Psychol 47: 211-218.

Braff D, Stone C, Callaway E, Geyer M, Glick I, Bali L (1978). Prestimulus effects on human startle reflex in normals and schizophrenics. Psychophysiology 15: 339-343.

Chitty K, Albrecht MA, Graham K, Kerr C, Lee JW, Iyyalol R et al (2014). Dexamphetamine effects on prepulse inhibition (PPI) and startle in healthy volunteers. Psychopharmacology 231: 2327-2337.

Chou HH, Talledo J, Lamb S, Thompson W, Swerdlow NR (2013). Amphetamine effects on MATRICS Consensus Cognitive Battery performance in healthy adults. Psychopharmacology 227: 165-176.

Cloninger CR (1987). A systematic method for clinical description and classification of personality variants. A proposal. Arch Gen Psychiatry 44: 573-588.

Goldberg TE, Bigelow LB, Weinberger DR, Daniel DG, Kleinman JE (1991). Cognitive and behavioral effects of the coadministration of dextroamphetamine and haloperidol in schizophrenia. Am J Psychiatry 148: 78-84.

Grobstein P (2005). Making the unconscious conscious: a bidirectional bridge between neuroscience/cognitive science and psychotherapy? Cortex 41: 663-668.

Hamidovic A, Dlugos A, Palmer AA, de Wit H (2010). Catechol-Omethyltransferase val158met genotype modulates sustained attention in both the drug-free state and in response to amphetamine. Psychiatr Genet 20: 85-92.

Hart AB, de Wit H, Palmer AA (2013). Candidate gene studies of a promising intermediate phenotype: failure to replicate. Neuropsychopharmacology 38: 802-816.

Hutchison KE, Swift R (1999). Effect of d-amphetamine on prepulse inhibition of the startle reflex in humans. Psychopharmacology 143: 394-400

Hutchison KE, Wood MD, Swift R (1999). Personality factors moderate subjective and psychophysiological responses to d-amphetamine in humans. Exp Clin Psychopharmacol 7: 493-501.

Kanabus M, Szelag E, Rojek E, Pöppel E (2002). Temporal order judgement for auditory and visual stimuli. Acta Neurobiol Exp (Wars) 62: 263-270.

Kim YH, Ko MH, Na SY, Park SH, Kim KW (2006). Effects of single-dose methylphenidate on cognitive performance in patients with traumatic brain injury: a double-blind placebocontrolled study. Clin Rehabil 20: 24-30.

Libet B (1985). Unconscious cerebral initiative and the role of conscious will in voluntary action. Behav Brain Sci 8: 529-566.

Libet B, Wright EW Jr, Feinstein B, Pearl DK (1979). Subjective referral of the timing for a conscious sensory experience: a functional role for the somatosensory specific projection system in man. Brain 102: 193-224.

Light GA, Zhang W, Joshi YB, Bhakta S, Talledo J, Swerdlow NR (2017). Single-dose memantine improves cortical oscillatory response dynamics in patients with schizophrenia. Neuropsychopharmacology 42: 2633-2639.

Mattay VS, Callicott JH, Bertolino A, Heaton I, Frank JA, Coppola R et al (2000). Effects of dextroamphetamine on cognitive performance and cortical activation. NeuroImage 12: 268-275.

Mattay VS, Goldberg TE, Fera F, Hariri AR, Tessitore A, Egan MF et al (2003). Catechol O-methyltransferase val158-met genotype and individual variation in the brain response to amphetamine. Proc Natl Acad Sci USA 100: 6186-6191.

McDonald BC, Flashman LA, Arciniegas DB, Ferguson RJ, Xing L, Harezlak J et al (2017). Methylphenidate and memory and attention adaptation training for persistent cognitive symptoms after traumatic brain injury: a randomized, placebo-controlled trial. Neuropsychopharmacology 42: 1766-1775.

Modell W, Hussar AE (1965). Failure of dextroamphetamine sulfate to influence eating and sleeping patterns in obese schizophrenic patients: clinical and pharmacological significance. JAMA 193: 275-278.

Nuechterlein KH, Green MF, Kern RS, Baade LE, Barch DM, Cohen JD et al (2008). The MATRICS Consensus Cognitive Battery, part 1: test selection, reliability, and validity. Am J Psychiatry 165: 203-213.

Norris H (1971). The action of sedatives on brain stem oculomotor systems in man. Neuropharmacology 10: 181-191.

Pietrzak RH, Snyder PJ, Maruff P (2010). Use of an acute challenge with d-amphetamine to model cognitive improvement in chronic schizophrenia. Hum Psychopharmacol 25: 353-358.

Preller KH, Ingold $\mathrm{N}$, Hulka LM, Vonmoos M, Jenni D, Baumgartner MR et al (2013). Increased sensorimotor gating in recreational and dependent cocaine users is modulated by craving and attention-deficit/hyperactivity disorder symptoms. Biol Psychiatry 73: 225-234.

Rapoport JL, Buchsbaum MS, Weingartner H, Zahn TP, Ludlow C, Mikkelsen EJ (1980). Dextroamphetamine. Its cognitive and behavioral effects in normal and hyperactive boys and normal men. Arch Gen Psychiatry 37: 933-943.

Shilling PD, Saint Marie RL, Shoemaker JM, Swerdlow NR (2008). Strain differences in the gating-disruptive effects of apomorphine: relationship to gene expression in nucleus accumbens signaling pathways. Biological Psychiatry 63: 748-758.

Slifstein M, van de Giessen E, Van Snellenberg J, Thompson JL, Narendran R, Gil R et al (2015). Deficits in prefrontal cortical and extrastriatal dopamine release in schizophrenia: a positron emission tomographic functional magnetic resonance imaging study. JAMA Psychiatry 72: 316-324.

Swerdlow NR (2011). Beyond antipsychotics: Pharmacologicallyaugmented cognitive therapies (PACTs) for schizophrenia. Neuropsychopharmacology 37: 310-311.

Swerdlow NR, Auerbach P, Monroe SM, Hartston H, Geyer MA, Braff DL (1993). Men are more inhibited than women by weak prepulses. Biol Psychiatry 34: 253-260.

Swerdlow NR, Bhakta S, Chou HH, Talledo JA, Balvaneda B, Light GA (2016a). Memantine effects on sensorimotor gating and mismatch negativity in patients with chronic psychosis. Neuropsychopharmacology 41: 419-430.

Swerdlow NR, Eastvold A, Karban B, Ploum Y, Stephany N, Geyer MA et al (2002). Dopamine agonist effects on startle and sensorimotor gating in normal male subjects: time course studies. Psychopharmacology 161: 189-201.

Swerdlow NR, Geyer MA, Braff DL (2001). Neural circuit regulation of prepulse inhibition of startle in the rat: Current knowledge and future challenges. Psychopharmacology 156: 194-215.

Swerdlow NR, Light GA, Cadenhead KC, Sprock J, Hsieh MH, Braff DL (2006a). Startle gating deficits in a large cohort of patients with schizophrenia: Relationship to medications, symptoms, neurocognition and level of function. Arch Gen Psychiatry 63: 1325-1335.

Swerdlow NR, Light GA, Sprock J, Calkins ME, Greene MF, Greenwood TA et al (2014). Deficient prepulse inhibition in 
schizophrenia detected by the multi-site COGS. Schizophr Res 152: 503-512.

Swerdlow NR, Light GA, Thomas ML, Sprock J, Calkins ME, Greene MF et al (2017). Deficient prepulse inhibition in schizophrenia in a multi-site cohort: Internal replication and extension. Schizophr Res (e-pub ahead of print).

Swerdlow NR, Shilling PD, Breier M, Trim RS, Light GA, Saint Marie RL (2012). Fronto-temporal-mesolimbic gene expression and heritable differences in amphetamine-disrupted sensorimotor gating in rats. Psychopharmacology 224: 349-362.

Swerdlow NR, Shoemaker JM, Bongiovanni MJ, Neary AC, Tochen LS, Saint Marie RL (2005). Reduced startle gating after D1 blockade: effects of concomitant D2 blockade. Pharmacol Biochem Behav 82: 293-299.

Swerdlow NR, Shoemaker JM, Kuczenski R, Bongiovanni MJ, Neary AC, Tochen LS et al (2006b). Forebrain D1 function and sensorimotor gating in rats: effects of D1 blockade, frontal lesions and dopamine denervation. Neurosci Lett 402: 40-45.

Swerdlow NR, Stephany N, Wasserman LC, Talledo J, Shoemaker J, Auerbach PP (2003). Amphetamine effects on prepulse inhibition across-species: replication and parametric extension. Neuropsychopharmacology 28: 640-650.

Swerdlow NR, Tarasenko M, Bhakta SG, Talledo J, Alvarez AI, Hughes EL et al (2016b). Amphetamine enhances gains in auditory discrimination training in adult schizophrenia patients. Schizophr Bull 43: 872-880.

Swerdlow NR, Weber M, Qu Y, Light GA, Braff DL (2008). Realistic expectations of prepulse inhibition in translational models for schizophrenia research. Psychopharmacol 199: 331-388.

Talledo JA, Sutherland Owens AN, Schortinghuis T, Swerdlow NR (2009). Amphetamine effects on startle gating in normal women and female rats. Psychopharmacology 204: 165-175.

Thomas ML, Green MF, Hellemann G, Sugar CA, Tarasenko M, Calkins ME et al (2017). Modeling deficits from early auditory information processing to psychosocial functioning in schizophrenia. JAMA Psychiatry 74: 37-46.

Wardle MC, Hart AB, Palmer AA, de Wit H (2013). Does COMT genotype influence the effects of d-amphetamine on executive functioning? Genes Brain Behav 12: 13-20.

Weafer J, Gorka SM, Hedeker D, Dzemidzic M, Kareken DA, Phan $\mathrm{KL}$ et al (2017). Associations between behavioral and neural correlates of inhibitory control and amphetamine reward sensitivity. Neuropsychopharmacology 42: 1905-1913.

Zuckerman M, Bone RN, Neary R, Mangelsdorff D, Brustman B (1978). What is the sensation seeker? Personality trait and experience correlates of the Sensation-Seeking Scales. J Consult Clin Psychol 39: 308-321.

Supplementary Information accompanies the paper on the Neuropsychopharmacology website (http://www.nature.com/npp) 